PHYSICAL REVIEW A 82, 061804(R) (2010)

\title{
Measuring nanomechanical motion with an imprecision below the standard quantum limit
}

\author{
G. Anetsberger, ${ }^{1}$ E. Gavartin, ${ }^{2}$ O. Arcizet,${ }^{1, \dagger}$ Q. P. Unterreithmeier, ${ }^{3}$ E. M. Weig, ${ }^{3}$ M. L. Gorodetsky, ${ }^{4}$ \\ J. P. Kotthaus, ${ }^{3}$ and T. J. Kippenberg ${ }^{1,2, *}$ \\ ${ }^{1}$ Max-Planck-Institut für Quantenoptik, Hans-Kopfermann-Straße 1, D-85748 Garching, Germany \\ ${ }^{2}$ Ecole Polytechnique Fédérale de Lausanne (EPFL), 1015 Lausanne, Switzerland \\ ${ }^{3}$ Fakultät für Physik and Center for NanoScience (CeNS), Ludwig-Maximilians-Universität (LMU), \\ Geschwister-Scholl-Platz 1, D-80539 München, Germany \\ ${ }^{4}$ Department of Physics, Moscow State University, Moscow RU-119899, Russia
}

(Received 12 March 2010; published 29 December 2010)

\begin{abstract}
We report a room-temperature measurement of nanomechanical motion with an imprecision below the standard quantum limit (SQL), using cavity-enhanced optical near fields. Fundamental thermodynamical cavity frequency fluctuation limits the total imprecision to $3 \mathrm{~dB}$ below the SQL at room temperature [corresponding to $(530 \mathrm{am} / \sqrt{\mathrm{Hz}})^{2}$ in absolute units] and is expected to reduce to negligible values at moderate cryogenic temperatures. Coupling strengths exceeding those required to reach the SQL by more than two orders of magnitude are achieved, allowing a shot-noise limited imprecision more than $10 \mathrm{~dB}$ below the SQL. The transducer, thus, in principle, allows access to the quantum backaction dominated regime, a prerequisite for exploring quantum backaction, measurement-induced squeezing, and obtaining sub-SQL sensitivity using backaction evading techniques.
\end{abstract}

DOI: 10.1103/PhysRevA.82.061804

PACS number(s): 42.50.Lc, 85.85.+j, 03.65.Ta, 72.70.+m

The development of nanomechanical oscillators [1,2] has enabled experiments ranging from precision measurements [3-5] to studies approaching the quantum regime of mechanical oscillators [6-8]. All these experiments rely on the sensitive detection of nanomechanical motion, and over the past few decades a variety of transducers have been developed. A natural scale for comparison, is their ability to achieve a measurement imprecision sufficient to resolve a mechanical oscillator's zero-point motion, which coincides with the sensitivity at the standard quantum limit (SQL) of continuous position measurement [9-12]. At the SQL the sensitivity of a displacement measurement $S_{x}^{\mathrm{SQL}}$ which arises from the balance of detector imprecision and measurement quantum backaction is equal to the quantum mechanical zero-point fluctuations (ZPF) of the nanomechanical oscillator under scrutiny. On resonance, these are given by $S_{x}^{\mathrm{ZPF}}=2 \hbar /\left(m \Omega_{\mathrm{m}} \Gamma_{\mathrm{m}}\right)$, where $m, \Gamma_{\mathrm{m}} / 2 \pi$, and $\Omega_{\mathrm{m}} / 2 \pi$ denote the effective mass, damping rate, and resonance frequency of the nanomechanical oscillator (single-sided spectra are used throughout this work).

Although, in principle, the measurement imprecision can be made arbitrarily small by increased coupling strengths between the transducer and the measured object (which leads to a corresponding increase in quantum backaction) [11], reaching an imprecision at the level of the ZPF (a prerequisite for reaching SQL sensitivity) remained an elusive goal for decades owing to both experimentally limited coupling strengths and excess classical noise. The lowest imprecision for nanomechanical motion (typically of the order $\mathrm{fm}^{2} / \mathrm{Hz}$ ) has been achieved by cryogenic transducers based on superconducting devices such as single-electron transistors [7,13,14], superconducting quantum interference devices [15], or quantum point contacts [16,17], and has approached the SQL [7,13-18], with the best value being $S_{x}=$

\footnotetext{
*tobias.kippenberg@epfl.ch

†Present address: Institut Néél, 25 rue des Martyrs, 38042 Grenoble, France.
}

$28 \times S_{x}^{\text {SQL }}$ [14]. The application of optical near fields [19,20] has subsequently allowed access to sub- $\mathrm{fm}^{2} / \mathrm{Hz}$ imprecision at room temperature $[19,20]$ and enabled an imprecision at the SQL [20]. Recently, using a cryogenic, a near quantum-limited microwave amplifier [21] has allowed the measurement of nanomechanical motion with an imprecision below the SQL, reaching $S_{x}=0.4 \times S_{x}^{\mathrm{SQL}}[22]$.

Here we show that using optical fields, sub-SQL imprecision for nanomechanical motion can also be achieved at room temperature, i.e., with strongly simplified experimental access. By using a cavity optomechanical near-field coupling scheme [20] with more than one order of magnitude improved optomechanical coupling coefficients combined with homodyne detection we reach a room-temperature imprecision of $S_{x}=(0.47 \pm 0.1) \times S_{x}^{\mathrm{SQL}}$ [23], launching only $1 \mu \mathrm{W}$ of optical input power. The transducer allows coupling strengths exceeding those required to reach the SQL by more than two orders of magnitude and a measured quantum (shot-noise) limited imprecision more than $10 \mathrm{~dB}$ below the SQL, i.e., $S_{x}^{\text {shot }}<0.1 \times S_{x}^{\mathrm{SQL}}$. Realizing this ultralow shot-noise limit at room temperature is inhibited by fundamental thermorefractive cavity frequency noise. Its magnitude is in excellent agreement with theory and is expected to decrease by $\geqslant 25 \mathrm{~dB}$ for operation below $30 \mathrm{~K}$, i.e., to a negligible level. The presented approach thereby paves the way to studying the quantum backaction (QBA) of radiation pressure [12,24] that ultimately enforces the SQL. Its observation in experiments with solid-state mechanical oscillators is still lacking, to date. Operating in the regime where QBA is the dominating contribution to sensitivity may enable measurement-induced squeezing [25] and leverage the use of backaction evading techniques [26,27].

Figure 1 shows the experimental setup. A small mode volume and high optical $Q\left(Q>10^{8}\right)$ silica toroid microresonator [28] [Fig. 1(a)] is fiber coupled using a fiber taper. The strained SiN nanomechanical oscillator [29,30] [Fig. 1(b), typical eigenfrequencies $\Omega_{\mathrm{m}} / 2 \pi \approx 10 \mathrm{MHz}$ ] interacts with the microresonator via the evanescent field [20,31] of its 

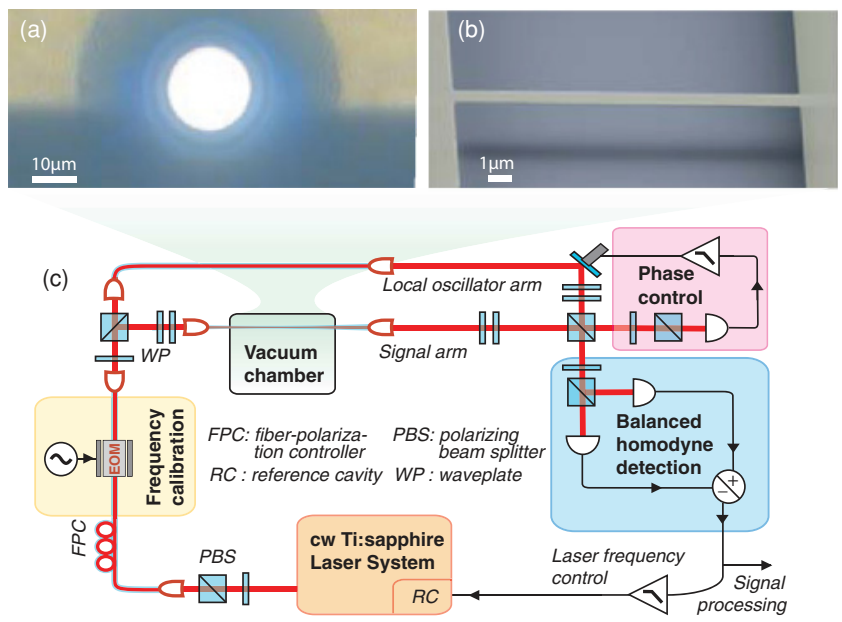

FIG. 1. (Color online) Optical and scanning electron micrographs of (a) a $16 \mu \mathrm{m}$ radius microresonator and (b) a $100 \mathrm{~nm} \times 500 \mathrm{~nm} \times$ $15 \mu \mathrm{m}$ strained SiN nanomechanical oscillator. (c) Schematic of the quantum-limited optical homodyne detection scheme. The samples are mounted in a vacuum chamber. A continuous wave (cw) titanium sapphire laser emitting at $\lambda=850 \mathrm{~nm}$ is fiber coupled.

optical whispering gallery modes. The interaction is described by the dispersive optomechanical coupling coefficient $g=$ $d \omega / d x$ and the reactive contribution $\gamma=d \kappa / d x$ (where $\omega / 2 \pi$ is the cavity resonance frequency and $\kappa / 2 \pi$ is the cavity energy decay rate). Both coupling coefficients scale inversely with wavelength $(\lambda)$ and cavity mode volume $(V)$ and exponentially depend on the separation $x$ between the microresonator and the nanomechanical oscillator, which is controlled using piezoelectric positioners. Both are calibrated statically and dynamically, as detailed elsewhere [20]. By employing a short wavelength $(\lambda=850 \mathrm{~nm})$ and a small mode volume $\left[V \sim(4 \mu \mathrm{m})^{3}\right]$, dispersive coupling coefficients $g / 2 \pi>200 \mathrm{MHz} / \mathrm{nm}$ can be achieved corresponding to a more than tenfold improvement compared to previous work [20]. For the respective vacuum optomechanical coupling rate $g_{0}=g \times x_{\mathrm{zpf}}$ one then obtains $g_{0} / 2 \pi>4 \mathrm{kHz}$ for typical values of $x_{\mathrm{zpf}}\left(x_{\mathrm{zpf}}=\sqrt{S_{x}^{\mathrm{ZPF}} \Gamma_{\mathrm{m}} / 4}\right)$. At the same time, the reactive coupling $\gamma$ is negligible with $\gamma \leqslant g / 20$ throughout this work.

Thus, the near-field interaction dispersively transduces the nanomechanical oscillator's Brownian noise displacement spectrum $S_{x}^{n}[\Omega]$ into frequency noise of the microresonator $S_{\omega}^{n}[\Omega]=g^{2} \times S_{x}^{n}[\Omega]$. The implemented measurement system consists of a shot-noise limited (for frequencies $\Omega>$ $500 \mathrm{kHz}$ ), cw titanium sapphire laser combined with a balanced homodyne interferometer, as shown in Fig. 1(c). The balanced homodyne detection enables quantum (shot-noise) limited measurements of the microresonator's frequency noise, as opposed to previous work [20] not limited by detector background noise even for very weak probe powers $(\sim 1 \mu \mathrm{W})$. We employ two individual feedback loops controlling the phase difference of the interferometer arms and locking the laser strictly to the line center of a microresonator mode, independently [cf. Fig. 1(c)]. Thus, dynamical backaction due to nonzero laser detuning is avoided, which could lead to linewidth-narrowing and artificially increased displacement

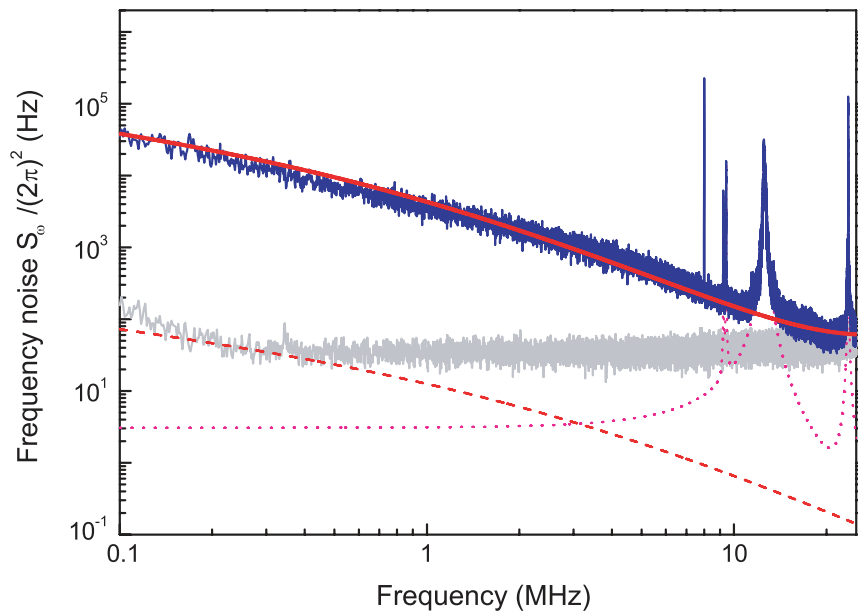

FIG. 2. (Color online) Frequency noise background of the microresonator. The measured equivalent frequency noise caused by shot noise (gray lower spectrum) lies far below the predominant broadband noise. The latter is due to thermorefractive cavity frequency noise according to Eq. (2) (thick red line, no adjustable parameters, also includes the measured shot noise). For a bath temperature of $30 \mathrm{~K}$, a $25 \mathrm{~dB}$ reduction of thermorefractive noise is expected (dashed line). The mechanical modes of the microresonator (dotted line) have negligible off-resonant contributions $(8 \mathrm{MHz}$ : calibration marker).

spectral densities of the nanomechanical oscillators [20]. This is particularly important, since due to the strongly increased optomechanical coupling, the threshold for the parametric instability [32,33] can be reached for only $400 \mathrm{nW}$ of input power.

Figure 2 shows a spectrum of the frequency noise of a microresonator (radius $R=18 \mu \mathrm{m}$ ) alone, without coupling to the nanomechanical oscillator. Owing to the quantumlimited detection scheme, the background of the measurement is only given by laser shot noise [20],

$$
S_{\omega}^{\text {shot }}[\Omega]=\frac{\hbar \omega}{P_{\text {in }}} \frac{\kappa^{2}}{8}\left(1+4 \Omega^{2} / \kappa^{2}\right) .
$$

By using sufficiently large input power $\left(P_{\text {in }} \gtrsim 5 \mu \mathrm{W}\right)$, its magnitude can be lowered far below both measured frequency noise sources: First, the intrinsic mechanical motion $S_{x}^{\mu}[\Omega]$ of the microresonator itself [34] causes the contribution $S_{\omega}^{\mu}[\Omega]=(\omega / R)^{2} \times S_{x}^{\mu}[\Omega]$. This source of frequency noise can be confined to narrow frequency bands by tuning the frequency and mechanical quality factor of the microresonator modes [34,35]. The second contribution is broadband and is given by the fundamental temperature fluctuations $S_{T}[\Omega]$ within the cavity mode volume [36] which lead to thermorefractive frequency noise $S_{\omega}^{\text {thr }}[\Omega]=[(\omega / n)(d n / d T)]^{2} \times S_{T}[\Omega]$ (where $n$ denotes the silica refractive index). For fundamental microtoroid modes, it is given by

$$
S_{\omega}^{\mathrm{thr}}[\Omega]=\left(\frac{\omega}{n} \frac{d n}{d T}\right)^{2} \frac{(16 \pi)^{1 / 3} k_{B} T^{2}}{V \rho C} \frac{\sqrt{\tau / \Omega}}{\left[1+(\Omega \tau)^{3 / 4}\right]^{2}},
$$

where $C$ and $\rho$ are the heat capacity and density of silica, respectively. The optical modes with angular mode number $\ell$ are approximated by Gaussian ellipses with semiaxes $b=0.77 R / \ell^{2 / 3}$ and $d=R^{3 / 4} r^{1 / 4} / \ell^{1 / 2}$ (where $r$ denotes the 


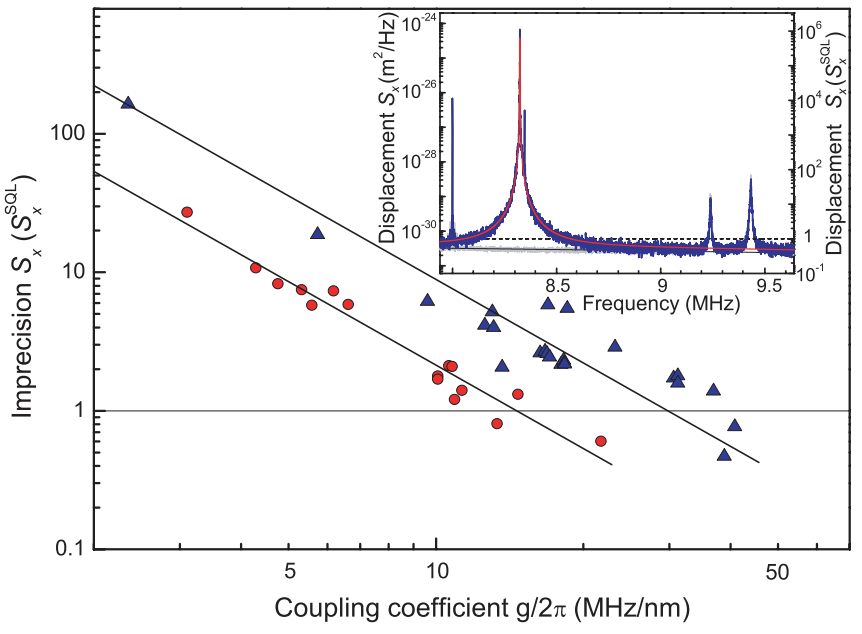

FIG. 3. (Color online) Optical measurement of nanomechanical motion with an imprecision below the SQL. The total measurement imprecision for an $8.3 \mathrm{MHz}$ nanomechanical oscillator $(4 \times$ $10^{-15} \mathrm{~kg}$ ), using $P_{\text {in }}=1 \mu \mathrm{W}$ (triangles) and $P_{\text {in }}=8 \mu \mathrm{W}$ (circles), reduces with increased optomechanical coupling $g / 2 \pi$ according to Eq. (3). Both power levels allow an imprecision below the SQL, achieving a best value of $S_{x}=0.47 \times S_{x}^{\mathrm{SQL}}$. Inset: Spectrum with the lowest imprecision at an input power of $1 \mu \mathrm{W}$ (calibration marker at $8 \mathrm{MHz}$, first-order in-plane mode of the string at $8.4 \mathrm{MHz}$, and mechanical modes of the optical microresonator at $>9 \mathrm{MHz}$ ).

minor toroid radius). The thermal cut-off time $\tau$ is given by $\tau^{-1}=(4 / \pi)^{1 / 3} D\left(b^{-2}+d^{-2}\right)$, where $D$ denotes the thermal diffusivity of silica. Good agreement between Eq. (2) and the measured data is found, as shown in Fig. 2 (no adjustable parameters). Thus, this source of frequency noise, which may also be of relevance for other nano-optomechanical transducers [19,37], is well understood.

Next, we place a SiN nanomechanical oscillator in the optical near field. The total measured spectrum $S_{x}[\Omega]$ thus consists of the following contributions:

$$
S_{x}[\Omega]=S_{x}^{n}[\Omega]+\frac{1}{g^{2}}\left(S_{\omega}^{\mathrm{shot}}[\Omega]+S_{\omega}^{\mathrm{thr}}[\Omega]+\frac{\omega^{2}}{R^{2}} S_{x}^{\mu}[\Omega]\right) .
$$

Increasing the optomechanical coupling $g$ (i.e., reducing the distance between the nanomechanical oscillator and the cavity) equally reduces all background noises, independent of their origin. This is shown in Fig. 3, where the measurement imprecision (i.e., the full background of the measurement) obtained for the first fundamental out-of-plane mode of a $30 \mu \mathrm{m} \times 700 \mathrm{~nm} \times 100 \mathrm{~nm}$ nanomechanical string is depicted as a function of $g / 2 \pi$. Increasing the optomechanical coupling allows one to obtain an imprecision $\sim 3 \mathrm{~dB}$ below the SQL both for 1 and $8 \mu \mathrm{W}$ input powers. This represents the first optical measurement of nanomechanical motion with an imprecision below the SQL. A closer inspection of the different background noise contributions reveals that the employed power levels are already far beyond the power level needed to reach the $\mathrm{SQL}\left(P_{\mathrm{SQL}}\right)$, thus falling in the regime where QBA should be the dominating contribution to sensitivity. In an ideal measurement with a lossless cavity, $P_{\mathrm{SQL}}$ is given by

$$
P_{\mathrm{SQL}} / \hbar \omega=\frac{(\kappa / 4)^{2}}{g^{2} S_{x}^{\mathrm{ZPF}}}\left(1+\frac{4 \Omega_{\mathrm{m}}^{2}}{\kappa^{2}}\right) .
$$

Equation (4) reveals that a photon flux of one photon per second allows the SQL to be reached if the ZPF modulate the cavity resonance by more than a quarter linewidth. Since experimentally available power levels are limited and high power levels, in addition, lead to thermal effects, minimizing $P_{\mathrm{SQL}}$ is generally desirable. Interestingly, the threshold power for the parametric oscillation instability $[32,33] P_{\text {th }}$, or alternatively, the power needed to cool the mechanical oscillator by a factor of 2, scales similarly: $P_{\mathrm{th}}=\left(4+\kappa^{2} / 4 \Omega_{\mathrm{m}}^{2}\right) \times P_{\mathrm{SQL}}$, with $P_{\mathrm{th}} \cong$ $4 \times P_{\mathrm{SQL}}$ in the resolved sideband regime [38]. Therefore, the figure of merit $P_{\mathrm{SQL}}$ describes a transducer's ability to both manipulate the mechanical oscillator via dynamical backaction and to reach the SQL. Owing to a tenfold improvement in optomechanical coupling, we were able to reduce $P_{\mathrm{SQL}}$ by two orders of magnitude compared to previous work [20]. Indeed, power levels of a few microwatts already correspond to $P_{\text {in }}>$ $100 \times P_{\mathrm{SQL}}$. Taking into account that in our measurements an impedance-matched cavity is used, the added noise quanta due to QBA are calculated to be $n_{\mathrm{QBA}} \sim 20$. However, these are currently not measurable due to the large thermal occupation of $\bar{n} \sim k_{B} T /\left(\hbar \Omega_{\mathrm{m}}\right)=7.5 \times 10^{5}$.

To nevertheless demonstrate operation far beyond $P_{\mathrm{SQL}}$, we analyze the different contributions to the measurement sensitivity. We emphasize that thermorefractive and shot-noise contributions can be measured individually in our experimental configuration by decoupling the nanomechanical oscillator

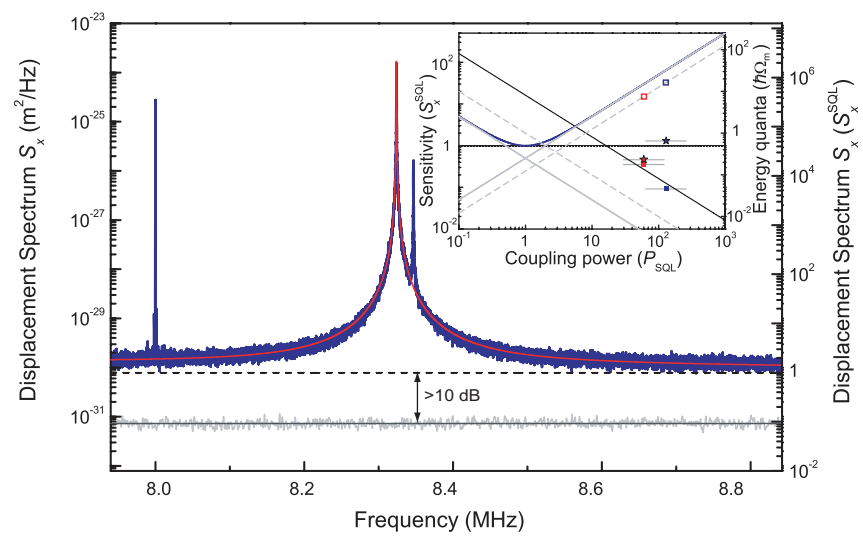

FIG. 4. (Color online) Shot-noise limited imprecision more than $10 \mathrm{~dB}$ below the SQL. The total measurement imprecision is approximately at the SQL. The shot-noise level (gray, lower line), however, lies $>10 \mathrm{~dB}$ lower at $S_{x}=0.09 \times S_{x}^{\mathrm{SQL}}$ or $(250 \mathrm{am} / \sqrt{\mathrm{Hz}})^{2}$. Inset: Sensitivity versus normalized optical input power. Full (dashed) gray lines mark shot noise and QBA for an ideal, lossless measurement (for an impedance-matched cavity). The solid blue line shows the total sensitivity. Stars mark the full imprecision for the spectra shown in Figs. 3 (inset) and 4 with different thermorefractive noise levels (owing to different optomechanical coupling). The measured shot-noise limited imprecision (squares) lies far below the value of the SQL, reaching $0.09 \times S_{x}^{\mathrm{SQL}}$ for $P_{\text {in }}=130 \times P_{\mathrm{SQL}}$. The corresponding QBA noise (empty squares) is estimated to contribute $n_{\mathrm{QBA}} \approx 20$ noise quanta. The full line (black) fits the measured shot-noise contribution. 
from the microresonator and detuning the measurement laser from cavity resonance, respectively. The inset of Fig. 3 shows a spectrum of the nanomechanical string with the lowest imprecision [at $S_{x}=0.47 \times S_{x}^{\mathrm{SQL}}$ or $(530 \mathrm{am} / \sqrt{\mathrm{Hz}})^{2}$ ], which is dominated by shot noise, whereas the thermorefractive contribution is small. This trace was recorded with $P_{\text {in }}=$ $1 \mu \mathrm{W}$. Increasing $P_{\text {in }}$ allows for a reduction in the shot-noise level and already an input power of $P_{\text {in }}=8 \mu \mathrm{W}$ enables a shot-noise limited imprecision of $0.09 \times S_{x}^{\mathrm{SQL}}$ (i.e., $>10 \mathrm{~dB}$ below the SQL, as shown in Fig. 4). Owing to the previously mentioned close connection between dynamical backaction and $P_{\mathrm{SQL}}$, small imperfections in the laser detuning can lead to strong dynamical backaction effects. Reliably ruling these out requires slightly reducing the optomechanical coupling when working with increased power levels, $g / 2 \pi=15 \mathrm{MHz} / \mathrm{nm}$ in the case of Fig. 4. Consequently, this higher power measurement is partly limited by thermorefractive noise $\left[S_{\omega}^{\mathrm{thr}}[\Omega]=\right.$ $(2 \pi 12 \sqrt{\mathrm{Hz}})^{2}$ at $\Omega / 2 \pi \sim 8.5 \mathrm{MHz}$, which as opposed to shot noise, does not reduce for higher laser power]. With the wellknown material parameters of silica, the thermorefractive noise according to Eq. (2) is suppressed by $\geqslant 25 \mathrm{~dB}$ when operating at $\leqslant 30 \mathrm{~K}$ instead of ambient temperature (see Fig. 2). Thus, thermorefractive noise will be negligible at low temperatures (without even taking into account the strongly increased mechanical $Q$ at low temperatures, increasing the SQL) and the demonstrated shot-noise limited imprecision $S_{x}<$ $0.1 \times S_{x}^{\mathrm{SQL}}$ may be realized. This represents a major advantage compared to even the most sensitive cryogenic transducers based on microwaves which are currently also limited by cavity frequency noise albeit operating at $130 \mathrm{mK}$ [22].

The inset of Fig. 4 shows the position of both measurements with respect to the SQL. The highest measurement power $(8 \mu \mathrm{W})$ corresponds to $P_{\text {in }}=130 \times P_{\mathrm{SQL}}$ and thus the contribution of QBA $\left(n_{\mathrm{QBA}} \approx 20\right)$ should be the dominating contribution to sensitivity, much larger than the imprecision caused by thermorefractive $\left(n_{\mathrm{thr}} \approx 0.5\right)$ and shot noise $\left(n_{\text {shot }}<0.1\right)$. However, in the current experiment this contribution is masked by the large number of thermal quanta $\left(\bar{n} \approx 7.5 \times 10^{5}\right)$. By using mechanical oscillators with narrower linewidths (e.g., $\Gamma_{\mathrm{m}} / 2 \pi=1 \mathrm{~Hz}$ as shown in Ref. [39] compared to $\Gamma_{\mathrm{m}} / 2 \pi \approx$ $300 \mathrm{~Hz}$ used here) and accessing the already demonstrated larger optomechanical coupling $g / 2 \pi>200 \mathrm{MHz} / \mathrm{nm}$ by improved stability of the measurement setup, the QBA could, however, be enhanced by a factor of $3 \times 10^{4}$ to $n_{\mathrm{QBA}}=$ $6 \times 10^{5}$, i.e., to values comparable to the thermal occupation, even at room temperature. This would allow quantum optomechanical experiments [24] at room temperature.

\section{ACKNOWLEDGMENTS}

T.J.K. acknowledges support from the DFG, the NIM Initiative, a Marie Curie Excellence Grant, the ERC Starting Grant SiMP and the NCCR of Quantum Photonics. E.M.W. and J.P.K. acknowledge support from the EC project QNEMS.
[1] K. L. Ekinci and M. L. Roukes, Rev. Sci. Instrum. 76, 061101 (2005).

[2] H. G. Craighead, Science 290, 1532 (2000).

[3] A. Cleland and M. Roukes, Nature (London) 392, 160 (1998).

[4] K. Jensen, K. Kwanpyo, and A. Zettl, Nature Nanotech. 3, 533 (2008).

[5] D. Rugar et al., Nature (London) 430, 329 (2004).

[6] K. C. Schwab and M. L. Roukes, Phys. Today 58, 36 (2005).

[7] M. D. LaHaye, O. Buu, B. Camarota, and K. C. Schwab, Science 304, 74 (2004).

[8] J. D. Teufel, J. W. Harlow, C. A. Regal, and K. W. Lehnert, Phys. Rev. Lett. 101, 197203 (2008).

[9] V. B. Braginskiǔ and Y. I. Vorontsov, Sov. Phys. Usp. 17, 644 (1975).

[10] V. B. Braginsky, Y. I. Vorontsov, and F. Y. Khalili, Pis'ma Zh. Eksp. Teor. Fiz. 27, 296 (1978).

[11] A. A. Clerk et al., Rev. Mod. Phys. 82, 1155 (2010).

[12] C. M. Caves, Phys. Rev. D 23, 1693 (1981).

[13] R. Knobel and A. Cleland, Nature (London) 424, 291 (2003).

[14] A. Naik et al., Nature (London) 193, 443 (2006).

[15] S. Etaki et al., Nature Phys. 4, 785 (2008).

[16] N. E. Flowers-Jacobs, D. R. Schmidt, and K. W. Lehnert, Phys. Rev. Lett. 98, 096804 (2007).

[17] M. Poggio et al., Nature Phys. 4, 635 (2008).

[18] C. A. Regal, J. D. Teufel, and K. W. Lehnert, Nature Phys. 4, 555 (2008)

[19] M. Eichenfield et al., Nature (London) 459, 550 (2009).

[20] G. Anetsberger et al., Nature Phys. 5, 909 (2009).

[21] M. Castellanos-Beltran et al., Nature Phys. 4, 929 (2008).
[22] J. Teufel et al., Nature Nanotech. 4, 820 (2009).

[23] All spectral densities are understood with $20 \%$ error bar.

[24] P. Verlot, A. Tavernarakis, T. Briant, P. F. Cohadon, and A. Heidmann, Phys. Rev. Lett. 102, 103601 (2009).

[25] C. Fabre et al., Phys. Rev. A 49, 1337 (1994).

[26] V. B. Braginsky and F. Y. Khalili, Quantum Measurement (Cambridge University Press, Cambridge, UK, 1992).

[27] J. Hertzberg et al., Nature Phys. 6, 213 (2009).

[28] T. J. Kippenberg, S. M. Spillane, and K. J. Vahala, Appl. Phys. Lett. 85, 6113 (2004).

[29] S. Verbridge et al., J. Appl. Phys. 99, 124304 (2006).

[30] Q. P. Unterreithmeier, E. M. Weig, and J. P. Kotthaus, Nature (London) 458, 1001 (2009).

[31] V. Braginsky and S. P. Vyatchanin, Quantum Optics, Experimental Gravity, and Measurement Theory (Plenum, New York, 1983).

[32] V. Braginsky, S. Strigin, and S. Vyatchanin, Phys. Lett. A 287, 331 (2001).

[33] T. J. Kippenberg, H. Rokhsari, T. Carmon, A. Scherer, and K. J. Vahala, Phys. Rev. Lett. 95, 033901 (2005).

[34] A. Schliesser et al., New J. Phys. 10, 095015 (2008).

[35] G. Anetsberger et al., Nature Photon. 2, 627 (2008).

[36] M. L. Gorodetsky and I. S. Grudinin, J. Opt. Soc. Am. B 21, 697 (2004).

[37] M. Li, W. H. P. Pernice, and H. X. Tang, Phys. Rev. Lett. 103, 223901 (2009).

[38] A. Schliesser et al., Nature Phys. 4, 415 (2008).

[39] S. S. Verbridge, H. G. Craighead, and J. M. Parpia, Appl. Phys. Lett. 92, 013112 (2008). 